\title{
Phenotypic and genotypic characterization of glucose-6-phosphate dehydrogenase deficiency in Argentina. Retrospective and descriptive study
}

\author{
Silvia Eandi Eberle, Biochemist ${ }^{a}$, Carolina Pepe, Biochemist ${ }^{a}$, Alejandro Chaves, B.S. ${ }^{a}$, \\ Fernando Aguirre, Biochemista ${ }^{a}$, Berenice Milanesio, Biochemista ${ }^{a}$, Diego Fernández, Biochemist ${ }^{a}$, \\ Vanesa Ávalos Gómez, M.D. ${ }^{a}$, Gabriela Sciuccati, M.D. ${ }^{a}$, Lilian A. Díaz, M.D. ${ }^{a}$, Andrea Candas, M.D. ${ }^{a}$, \\ Carolina Cervio, M.D. ${ }^{a}$, Mariana Bonduel, M.D. ${ }^{a}$ and Aurora Feliu Torres, M.D. ${ }^{a}$
}

\begin{abstract}
Glucose-6-phosphate dehydrogenase deficiency is an erythrocyte enzyme disorder caused by mutations in the G6PD gene, which has an X-linked inheritance. Here we analyze the clinical and laboratory characteristics of 24 subjects with G6PD deficiency over 25 years. Their median age at diagnosis was 10.2 years (range: $0.6-56.4$ ). No symptoms were observed in $54.2 \%$ of patients, whereas $25 \%$ had chronic non-spherocytic hemolytic anemia; $12.5 \%$, neonatal jaundice and postinfectious hemolytic anemia; and $8.3 \%$, acute hemolytic anemia after ingestion of fava beans. The 24 studied patients had variants that had been previously described in the bibliography. The clinical characteristics observed here were consistent with the variants found. A total of 21 women from the maternal line of affected subjects were identified as deficiency carriers using molecular biology techniques, so they received the corresponding genetic counseling.

Key words: inborn errors of metabolism, glucose-phosphate dehydrogenase, molecular diagnostic techniques.
\end{abstract}

http: / / dx.doi.org/ 10.5546/ aap.2019.eng.267

To cite: Eandi Eberle S, Pepe C, Chaves A, Aguirre F, et al. Phenotypic and genotypic characterization of glucose-6-phosphate dehydrogenase deficiency in Argentina. Retrospective and descriptive study. Arch Argent Pediatr 2019;117(4):267-270.

a. Hospital de Pediatría “Prof. Dr. Juan P. Garrahan.

E-mail address:

Silvia Eandi Eberle, Biochemist: seandi@garrahan.gov.ar

Funding: None.

Conflict of interest: None.

Received: 9-5-2018

Accepted: 2-18-2019

\section{INTRODUCTION}

Glucose-6-phosphate dehydrogenase (G6PD) catalyzes the first reaction in the pentose phosphate pathway, which generates reduced nicotine adenine dinucleotide phosphate $(\mathrm{NADPH})$, the main provider of reducing power to all cells. The NADPH generated is critical to prevent oxidative damage by maintaining intraerythrocytic concentrations of glutathione. ${ }^{1}$ G6PD deficiency affects approximately 500 million people worldwide and is the most common erythrocyte enzyme pathology. Its area of distribution is consistent with that of malaria., ${ }^{2,3}$

G6PD mutations (Xq28) determine protein variants with different enzyme activity levels, associated with a wide range of clinical and biochemical phenotypes.

The clinical manifestations of G6PDdeficient subjects are highly variable, from being asymptomatic to having neonatal jaundice, acute hemolytic anemia (AHA) triggered by infectious agents, drugs and/or fava bean ingestion, or chronic non-spherocytic hemolytic anemia (CNSHA). As per the classification by the World Health Organization (WHO), the variants leading to CNSHA correspond to class I, whereas those resulting in oxidative stress-induced hemolytic events (infections, drugs or fava bean ingestion) are class II or III. Class IV variants display a normal enzyme activity. Luzzato and Poggi have proposed to merge classes II and III because all these variants have an enzyme activity of less than $30 \% .^{2-4}$

The objective of this study was to describe the clinical and the conventional and molecular laboratory characteristics of subjects with G6PD deficiency diagnosed at the Department of Hematology and Oncology of Hospital de Pediatría "Prof. Dr. Juan P. Garrahan".

\section{PATIENTS AND METHODS}

This was a retrospective, descriptive, and longitudinal study that included 24 subjects 
(23 males and 1 female) diagnosed with G6PD deficiency from 14 unrelated families seen at the hospital between March 1991 and October 2016. The cases of 6 male subjects had been previously reported. ${ }^{5}$

Diagnosis was based on testing for erythrocyte pathology done in affected subjects and their families. The study included clinical assessment, complete blood count (Sysmex XS 800i, Sysmex Corporation, Kobe, Japan), peripheral blood smear, reticulocyte count, hemoglobin electrophoresis at alkaline $\mathrm{pH}$ on agarose gels, capillary electrophoresis (Sebia, Lisses, Évry, France), hemoglobin A2 by anion exchange chromatography (Helena), hemoglobin $\mathrm{F}$ by alkali denaturation test, erythrocyte osmotic resistance test, and iron metabolism. G6PD deficiency was diagnosed based on Brewer's test and enzyme activity levels (Trinity Biotech). ${ }^{4}$

For the molecular test, deoxyribonucleic acid (DNA) was isolated in total peripheral blood leukocytes using the saline precipitation method. The presence of point mutations and / or small insertions-deletions in the G6PD gene was assessed using a polymerase chain reaction (PCR); an automatic sequencer (ABI PRISM ${ }^{\circledR} 3130$ ) and the primers and strategy described before were used for such sequencing. ${ }^{6}$

The study was approved by the Research Review Committee of Hospital de Pediatría "Prof. Dr. Juan P. Garrahan." Patients and/or their parents signed the corresponding consent at the time of the first test for erythrocyte pathology.

\section{RESULTS}

During the study period, 46 subjects were diagnosed with G6PD deficiency (45 males and 1 female). Genotypic characterization was possible in 24 subjects only; their median age was 10.2 years (range: 0.6-56.4). Table 1 describes the characteristics of the study population.

Six patients (\#1-\#6), from 4 unrelated families, had CNSHA with an enzyme activity of less than $10 \%$ and the molecular variants described as class I in the bibliography. Patients \#7, \#8, and \#9 had neonatal jaundice, transfusion requirement due to infectious disease, and an enzyme activity of less than $10 \%$ associated with the Mediterranean variant described as class II in the bibliography. Nine patients from 6 families had the $A^{-202}$ molecular variant (class III) and an enzyme activity between $10 \%$ and $30 \%$. Seven patients in this group (\#10-\#16) were asymptomatic and G6PD deficiency was detected in the context of family testing for erythrocyte pathology. The other 2 patients (\#17 and \#18) were siblings with different clinical manifestations: one was asymptomatic and the other had a hemolytic event after the ingestion of fava beans. In the group of 5 patients with the $\mathrm{A}^{-968}$ variant, 2 asymptomatic siblings were detected by a positive Brewer's test in the context of family testing for

TABLE 1. Clinical and laboratory characteristics

\begin{tabular}{|c|c|c|c|c|c|c|}
\hline \#Pt (Fly) & Sex & Class & EA $\%$ & Clinical & NP_001035810.1 mutation & Variant \\
\hline$\# 1(1)$ & $\mathrm{M}$ & I & $<10$ & CNSHA & p.(Ser332Phe) & Quilmes \\
\hline$\# 2(2)$ & M & I & $<10$ & CNSHA & p.(Pro409Gln) & Merlo \\
\hline$\# 3, \# 4, \# 5(3)$ & M & I & $<10$ & CNSHA & p.(Phe381Ile) & New York \\
\hline$\# 6(4)$ & M & I & $<10$ & CNSHA & p.(Arg387His) & Beverly Hills \\
\hline$\# 7$ (5) & M & II & $<10$ & NJ AHA & & \\
\hline$\# 8(6)$ & M & II & $<10$ & NJ AHA & p.(Ser188Phe) & Mediterránea \\
\hline$\# 9(7)$ & M & II & $<10$ & NJ AHA & & \\
\hline$\# 10(8)$ & M & III & $10-30$ & ASX & & \\
\hline$\# 11, \# 12, \# 13$ (9) & M & III & $10-30$ & ASX & & \\
\hline \#14 (10) & M & III & $10-30$ & ASX & & \\
\hline \#15 (11) & M & III & $10-30$ & ASX & p.(Val68Met, Asn126Asp) & $\mathrm{A}^{-202}$ \\
\hline \#16 (12) & M & III & $10-30$ & ASX & & \\
\hline \#17 (13) & M & III & $10-30$ & ASX & & \\
\hline \#18 (13) & M & III & $10-30$ & AHA & & \\
\hline$\# 19, \# 20$ (14) & M & III & $10-30$ & ASX & & \\
\hline \#21 (12) & M & III & $10-30$ & AHA & p.(Asn126Asp, Leu323Pro) & $\mathrm{A}^{-968}$ \\
\hline$\# 22, \# 23$ (12) & M & III & $10-30$ & ASX & & \\
\hline$\# 24(12)$ & $\mathrm{F}$ & III & $10-30$ & ASX & p.(Val68Met, Leu323Pro) & $\mathrm{A}^{-202} / \mathrm{A}^{-968}$ \\
\hline
\end{tabular}

Fly: family; M: male; F: female; EA: enzyme activity; ASX: asymptomatic; CNSHA: chronic non-spherocytic hemolytic anemia; NJ: neonatal jaundice; AHA: acute hemolytic anemia; NP: reference number for the protein sequence. 
hemoglobinopathy. The other 3 patients (\#21, $\# 22, \# 23)$ with the $\mathrm{A}^{-968}$ variant were siblings. The index case (\#21) had a hemolytic event after the ingestion of fava beans, while the siblings were asymptomatic (\#22,\#23). The last patient (\#24) was an asymptomatic female with a positive Brewer's test and double heterozygous for the $\mathrm{A}^{-968} / \mathrm{A}^{-202}$ variants. She was the mother of 4 patients, 3 of which had the $A^{-968 w}$ variant $(\# 21$, $\# 22$, and \#23) and 1 with the $A^{-202}$ variant (\#16).

Based on sequencing and identification of the molecular variant involved, all women available in the maternal line were tested (mothers, sisters, aunts, and cousins). Thus, out of 29 studied women from 9 families, 21 were identified as heterozygous carriers.

\section{DISCUSSION}

G6PD is a housekeeping enzyme that is vital for cells; an absolute absence of enzyme activity would be incompatible with life.

G6PD catalyzes the first step in the pentose phosphate pathway. This metabolic pathway generates $\mathrm{NADPH}$, which helps to maintain an adequate reduced cellular environment, especially in erythrocytes.

G6PD deficiency is widely heterogeneous in terms of clinical and molecular manifestations. Worldwide, more than 200 variants of the G6PD gene have been reported in association with very mild symptoms, as observed in most patients with the A- variant, or severe symptoms, as seen in patients with the Mediterranean variant and severe hemolytic anemia or in those with the Guadalajara variant and CNSHA. ${ }^{2}$

In our series of 24 patients who had conventional and molecular testing, a correlation was established among clinical manifestations, enzyme activity, and molecular defect. In patients with CNSHA and an enzyme activity $<10 \%$, molecular testing identified 4 variants that corresponded to the WHO class I; of these, 2 (Merlo and Quilmes) have been recently described by our laboratory. ${ }^{7}$

Three of the variants observed (Merlo, New York, and Beverly Hills) are located in exon 10 and affect the amino acids involved in dimerization; therefore, they alter a critical step for the presence of catalytic activity. These mutations break the contact between 2 subunits or disrupt the interface structure by introducing amino acids with a different electrical charge or residues of different sizes. ${ }^{7,8}$

In the Quilmes variant, the substitution of
Ser332Phe may result in a conformational change in the loop of access to the active site, which would affect enzyme activity by modifying the binding to the glucose-6-phosphate (G6P) substrate. ${ }^{7}$

The Mediterranean variant, which was present in 3 patients, is located in exon 6 . The new coded amino acid increases the enzyme affinity for G6P. This feature may explain why, although these were severely deficient patients, they did not have CNSHA and were considered class II. ${ }^{2}$

The $\mathrm{A}^{-202}$ variant is the result of 2 missense changes affecting exons 4 and 5 . The threedimensional molecular test shows that the affected amino acids get closer in such a way that both residues are, spatially speaking, really close to each other. Based on this, such changes would play a synergistic role and cause deficiency due to the disruption in the protein structure, thus affecting the coenzyme domain. ${ }^{9}$

Lastly, the $\mathrm{A}^{-968}$ variant shows a multiple, missense-like change affecting exons 5 and 9. This variant determines the Asn126Asp and Leu323Pro amino acid substitutions. It has been proposed that, as a result of such molecular changes, both enzyme activity and stability are affected. The most predominant effect is probably due to the Leu323Pro substitution in the characterization of the $\mathrm{A}^{-968}$ variant. ${ }^{10}$

To conclude, confirming that there was a phenotype-genotype correlation allowed us to design a molecular testing algorithm for the G6PD gene based on the clinical characteristics of tested patients. In addition, thanks to the identification of a molecular alteration in males with G6PD deficiency, it was possible to provide genetic counseling to the women from the different families.

\section{REFERENCES}

1. Cappellini MD, Fiorelli G. Glucose-6-phosphate dehydrogenase deficiency. Lancet. 2008; 371(9606):64-74.

2. Luzzatto L, Poggi V. Glucose-6-phosphate dehydrogenase deficiency. In: Orkin SH, Nathan D, Fisher DE, Ginsburg $\mathrm{D}$, et al (eds.). Nathan and Oski's Hematology of Infancy and Childhood. $8^{\text {th }}$ ed. Philadelphia: Saunders Elsevier; 2015. Pages.609-29.

3. Beutler E. G6PD: population genetics and clinical manifestations. Blood Rev. 1996; 10(1):45-52.

4. Roper D, Layton M. Investigation of the hereditary haemolyticanaemias:membraneand enzymeabnormalities. In: Lewis SM, Bain BJ, Bates I(eds.). Dacie and Lewis' Practical Haematology. $10^{\text {th }}$ ed. Philadelphia: Churchill Livingston; 2006.Pages.205-37.

5. EandiEberle S, GarcíaRosolen N, Urtasum C, Sciucati G, et al. Deficiencia de glucosa-6-fosfato deshidrogenasa. Serie de casosclínicos. Arch Argent Pediatr. 2011; 109(4):354-7.

6. Poggi V, Town M, Foulkes NS, Luzzatto L. Identification of a single base change in a new human mutant glucose- 
6-phosphate dehydrogenase gene by polymerase-chain reaction amplification of the entire coding region from genomic DNA. Biochem J. 1990; 271(1):157-60.

7. Chaves A, Eberle SE, Defelipe L, Pepe C, et al. Two novel DNA variants associated with glucose-6-phosphate dehydrogenase deficiency found in Argentine pediatric patients. Clin Biochem. 2016; 49(10-11): 808-10.

8. Lee J, Park J, Choi H, Kim J, et al. Genetic Profiles of KoreanPatients With Glucose-6-Phosphate Dehydrogenase Deficiency. Ann Lab Med. 2017; 37(2):108-16.
9. Naylo CE, Rowland P, Basak AK, Gover S, et al. Glucose 6-Phosphate Dehydrogenase Mutations Causing Enzyme Deficiency in a Model of the Tertiary Structure of the Human Enzyme. Blood. 1996; 87(7):2974-82.

10. Ramírez-Nava EJ, Ortega-Cuellar D, Serrano-Posada H, González-Valdez A, et al. Biochemical Analysis of Two Single Mutants that Give Rise to a Polymorphic G6PD A-Double Mutant. Int J Mol Sci. 2017; 18(11):E2244. 